\title{
A PROSPECTIVE STUDY OF COMPLICATIONS IN EMERGENCY LAPAROTOMY
}

\author{
G. Raja Bily Graham¹, V. Vijayabhasker ${ }^{2}$ \\ 1 Professor, Department of General Surgery, Government Chengalpattu Medical College. \\ ${ }^{2}$ Assistant Professor, Department of General Surgery, Melmaruvathur Adhiparasakthi Institute of Medical Sciences and Research, \\ Tamilnadu.
}

\section{ABSTRACT}

Laparotomy or celiotomy is opening of the peritoneal cavity. Since the emergence of surgery, it is one of the common surgical procedure done for elective and emergency gastrointestinal and sometimes for non-gastrointestinal diseases and it is not always free from complications. The common complications associated with laparotomies are Bleeding, Haematoma, Fever, Surgical Site Infection, Wound dehiscence, Burst abdomen, Ileus, DVT, Subdiaphragmatic abscess, Stitch abscess, Enterocutaneous fistula, Hernia, Intestinal Obstruction, Atelectasis. These complications are more common in emergency laparotomy than with elective laparotomies. These complications pose a significant problem and add significantly to the morbidity and mortality associated with the proce dure itself. In order to know the pattern of incidence of various complications and to device a protocol for the prevention and the effective management of the post emergency laparotomy complications in a peripheral teaching hospital with limited resources, a prospective study has been formulated including 100 willing patients in the age group of 13 years to 70 without any significant comorbid medical illnesses with acute abdomen who underwent emergency laparotomy or various causes like perforation peritonitis, blunt/penetrating abdominal injury, intestinal obstruction, etc. Laparotomy procedure was standardized for all patients like midline vertical incision was used, same suture materials were used for all patients and abdominal drain was used for all patients. The complications were evaluated and managed appropriately. The data collected were analysed statistically.

\section{KEYWORDS}

Laparotomy, Perforation, Peritonitis, Wound Dehiscence.

HOW TO CITE THIS ARTICLE: Graham GRB, Vijayabhasker V. A prospective study of complications in emergency laparotomy. J. Evolution Med. Dent. Sci. 2016;5(12):513-519, DOI: 10.14260/jemds/2016/118

\section{INTRODUCTION}

Emergency laparotomy is required for major intra-abdominal haemorrhage spontaneous or due to abdominal trauma and for infective, ischemic and obstructive condition in which gastric intestinal wall as a barrier is breached or threatened.

Exploratory laparotomy is carried out in conditions where the need for an operation is recognized, but where a definite diagnosis can be made only until the abdomen is opened.

Emergency laparotomy is a high risk procedure, which involves making an incision preferably in the midline to provide access to abdominal cavity and is associated with significant morbidity and mortality.

Term also describes procedure for which the clinical presentation, pathology, anatomical site, perioperative management vary considerably and immediate intervention is warranted to save the life of the patient.

\section{OBJECTIVES OF STUDY}

A prospective study of emergency laparotomies among patients attending Chengalpattu Medical College with acute abdomen and to know about its complications and management in our setup.

Financial or Other, Competing Interest: None.

Submission 08-11-2015, Peer Review 20-11-2015,

Acceptance 24-11-2015, Published 10-02-2016.

Corresponding Author:

Dr. G. Raja Bily Graham,

No. 53, Vedhachalam Nagar,

Chengalpattu-603001,

Kanchipuram District, Tamilnadu.

E-mail: grbgraham@gmail.com

DOI: $10.14260 /$ jemds/2016/118

\section{MATERIALS AND METHODS}

This prospective study was carried out in our institution over a period of 2 years from $1^{\text {st }}$ October 2011 to $30^{\text {th }}$ September 2013. All patients attending our institution found to have acute abdomen by clinical evaluation are included in the study.

Data regarding name, age, inpatient number, gender, relevant history, physical status, investigations such as haemoglobin\%, complete blood count, TC, DC, blood sugar, urea, creatinine, electrolytes, serum proteins, blood grouping and $\mathrm{Rh}$ typing, chest radiograph, X-ray abdomen erect, ultrasound abdomen and pelvis, CT scan abdomen and pelvis, anatomical site of surgery, type of incision used, ASA category, diagnosis, preoperative antibiotics, operative procedure done, wound classification, duration of surgery, length of hospital stay, postoperative complications such as were tabulated and recorded in the prescribed format. Postoperative complication given emphasis were:

1. Bleeding.

2. Haematoma.

3. Fever.

4. SSI.

5. Wound dehiscence.

6. Burst abdomen.

7. Ileus.

8. DVT.

9. Subdiaphragmatic abscess.

10. Stitch abscess.

11. Enterocutaneous fistula.

12. Hernia.

13. Intestinal obstruction.

14. Atelectasis.

15. MI.

16. Dementia, etc. 
Cases are selected by following Inclusion and Exclusion Criteria

\section{Inclusion Criteria}

- All acute abdomen (Blunt injury, peritonitis, bowel strangulation/obstruction) cases attending our institution.

- $\quad$ Age $>13$ yrs. and $<70$ yrs.

- Laparotomy, which were approached by midline incision.

\section{Exclusion Criteria}

- Paediatric age $<12$ yrs.

- Pregnant women.

- Lactating mothers.

- Female patients with gynaecological disease.

- Laparotomy, which were approached by other than midline incision.

- Acute abdomen managed by conservative methods.

- Prolene 1-0 was used universally for rectus closure by continuous locking sutures; 2-0 Vicryl was the absorbable suture, which was used for anastomosis. Urinary catheterization was routinely done for all cases. Abdominal tube drain was routinely placed one in the sub-Morrison and another in the pelvic cavity as warranted by the diagnosis. Prolene 3-0 used for approximation of skin by interrupted sutures.

\section{OBSERVATION AND RESULTS}

\section{Age Incidence}

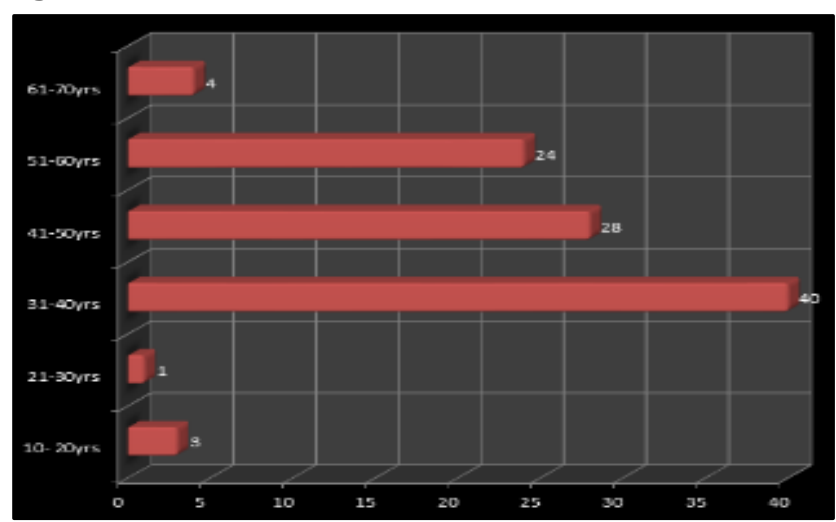

In our study, most numbers of laparotomies were done between the 31 and 40 years to be followed by 41 to 50 years. Perforation was the most common in these cases, whereas malignant obstruction was more common in the 60 plus group. In 12 to 20 years and 20 to 30 years, appendicular pathology was more common.

\section{Sex Incidence}

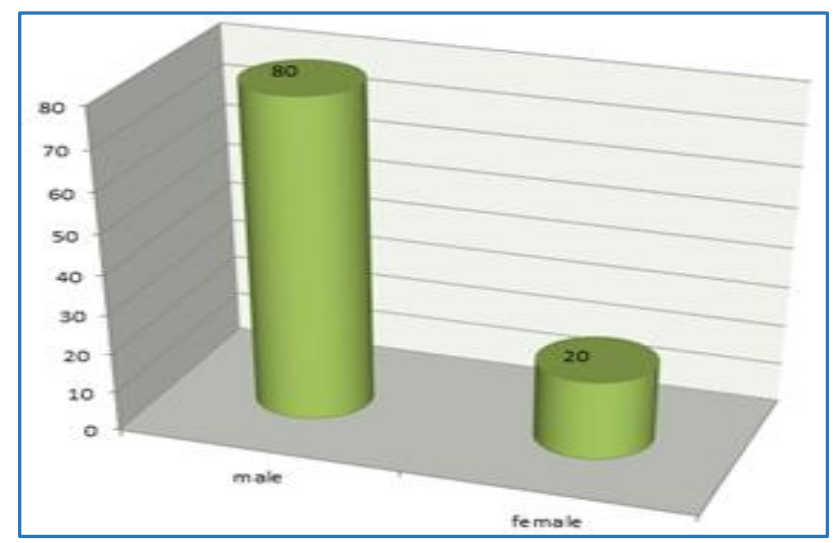

In our study of 100 emergency laparotomy cases, the males outnumbered the females by a ratio of $4: 1$. Acute abdomen was more common in male due to the fact of consumption of alcohol and blunt injury due to their large outdoor activities. Duodenal perforation was more common in males, whereas in females, intestinal obstruction was more common due to more prevalence of family planning surgery in them. Appendicular pathology followed next in frequency.

\section{Incidence}

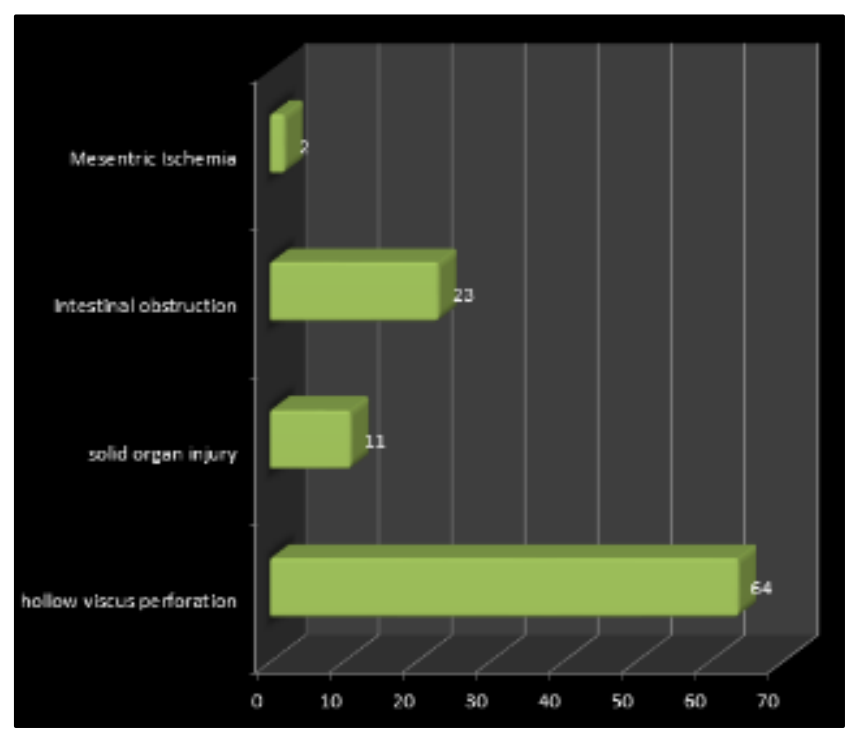

In our study of 100 cases, hollow viscus perforation was the most common cause for acute abdomen and constituted $64 \%$. Second most common cause was followed by intestinal obstruction [23\%] to be followed with solid organ injury [11\%]. Mesenteric ischemia constituted the least [2\%] cause for emergency laparotomy in our setup.

\section{Specific Aetiology of Acute Abdomen}

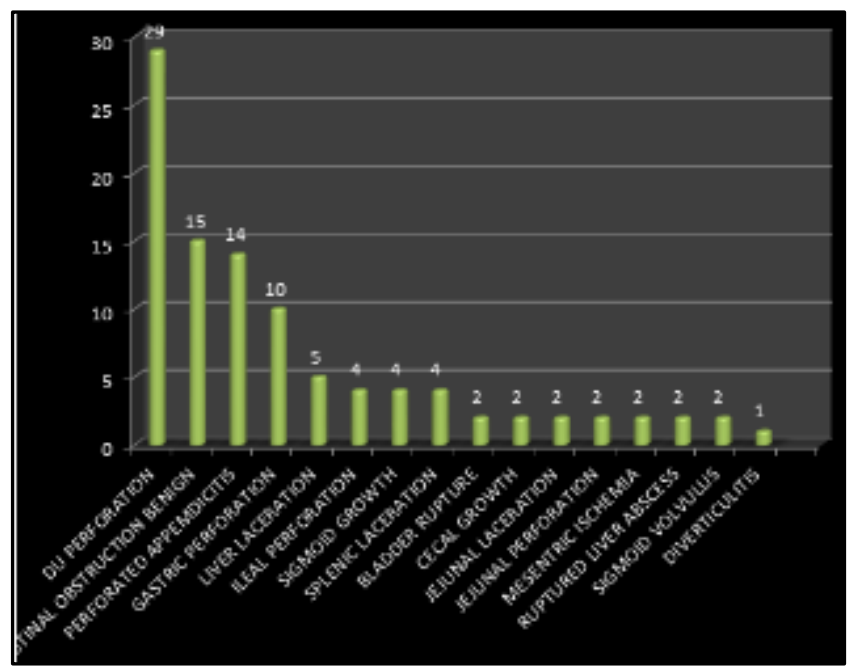

DU perforation was the most common cause of acute abdomen with a percentage of $29 \%$. Intestinal obstruction was the second most common cause of acute abdomen needing emergency laparotomy with a percentage of $23 \%$ and frequency much higher in females. Perforated appendicitis constituted 14\%, which were explored by lower midline incision. Perforation of stomach constituted $10 \%$, liver 
laceration constituted $5 \%$ and splenic laceration requiring splenectomy constituted $4 \%$. In the remaining $15 \%$, emergency laparotomy was indicated for rare causes such as jejunal laceration/perforation, ileal perforation, bladder rupture, diverticulitis, sigmoid volvulus and mesenteric ischemia.

\section{Outcome-Complications}

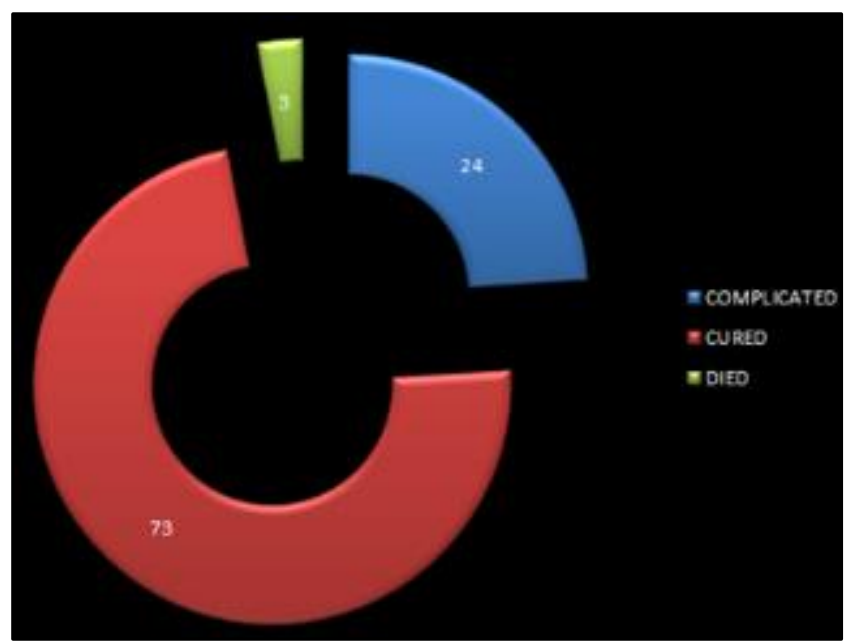

In the study of 100 patients, 73 patients underwent laparotomy for specific cause and got discharged without any complications and the remaining 27 had postoperative complications. $^{1}$ Out of these 27 cases, 3 expired postoperatively due to cardiovascular problem [MI]; 24 patients developed complications in the postoperative period, which were dealt appropriately.

\section{Complications}

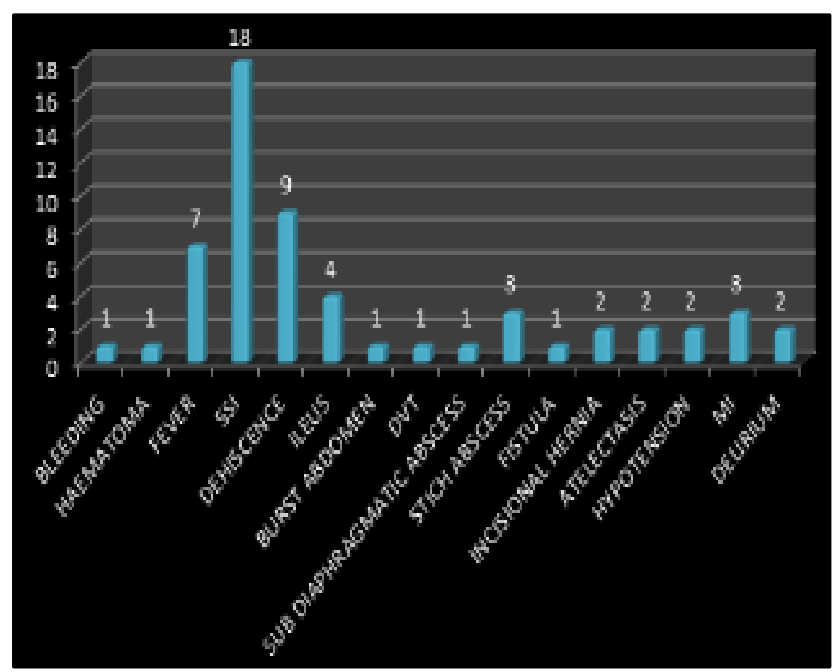

SSI was the most common postoperative complication in our setup accounting for $66 \%$ of the cases. Apart from being the most common cause of postoperative complication, SSI also predisposed the patient to other complications such as fever, wound gaping and burst abdomen in the immediate postoperative period and incisional hernia.1,2,3,4 in the late postoperative period. Wound dehiscence. 5,6 was the next most common with $33 \%$ and it was always predisposed by SSI. $7,8,9,10,11$

Fever constituted $26 \%$ and was present in most patient with SSI and in patient with subdiaphragmatic abscess. Stitch abscess and myocardial ischemia were fourth in frequency with $22 \%$ and mortality was $100 \%$ in the myocardial ischemia group. ${ }^{12,13}$ Stitch abscess was explored after 3 months and Prolene was found to be responsible for the abscess. Paralytic ileus was present in $15 \%$, which responded to conservative management. ${ }^{14,15}$

Atelectasis was the most common pulmonary complication and delirium was the most common central nervous system complication. Hypotension was the second most common complication requiring immediate management in the postoperative period and the risk factor was preoperative hypotension and epidural anaesthesia.

Total percentage of complications exceeds $100 \%$, since a single complication predisposes to other related complications; for example, SSI predisposes to wound dehiscence which in turn predisposes to burst abdomen or incisional hernia later. Similarly, SSI may present with fever. $1,2,3,4$

Bleeding from wound, haematoma, DVT.16 subdiaphragmatic abscess and burst abdomen were the least causes and two condition subdiaphragmatic abscess and burst abdomen were re-explored. Burst abdomen was managed by placing warm saline packs and immediate re-exploration at operation theatre.

\section{Duodenal and Gastric perforation surgery complications}

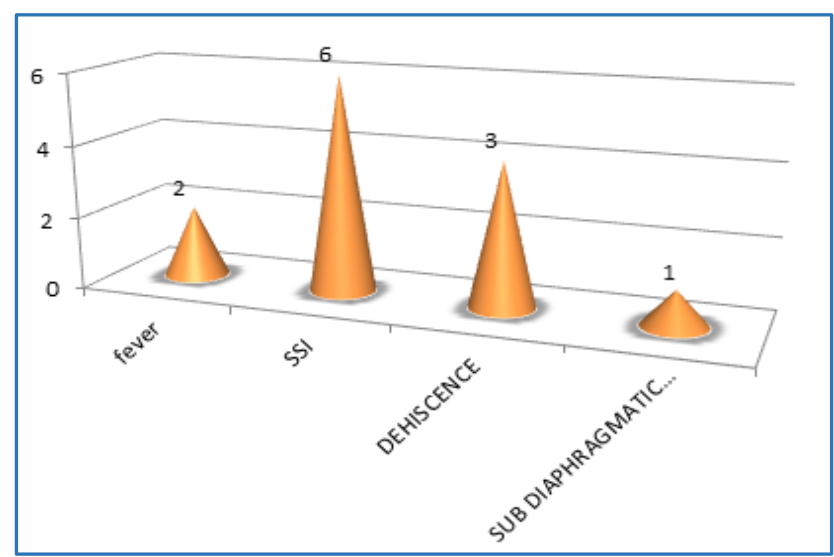

Out of the 39 duodenal and gastric perforations, alcohol was the predominant predisposing factor and it also constituted for postoperative delirium. In the course of management, 6 patients developed SSI, 3 developed dehiscence, 2 developed fever and 2 developed delirium due to alcohol withdrawal. One patient developed subdiaphragmatic abscess. Out of all these complications, only subdiaphragmatic abscess required immediate laparotomy. Other complications were treated conservatively.

Subdiaphragmatic abscess was reopened and pus from both the right and left subdiaphragmatic space was drained with warm saline and antibiotic wash. Abdomen closed by placing two abdominal drain. Postoperatively, patient improved gradually and was discharged on day 21 . 


\section{Wound Classification}

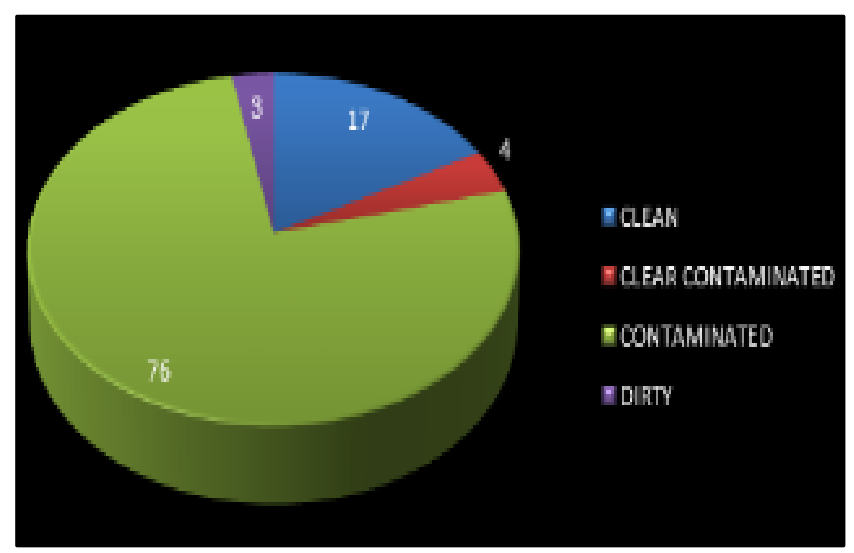

Out of 100 cases, percentage of wound classification were as follows - $17 \%$ of cases were clean, $4 \%$ were clear contaminated, $76 \%$ were contaminated and $3 \%$ were dirty.

Incidence of SSI in clean, clean contaminated, contaminated and dirty wound

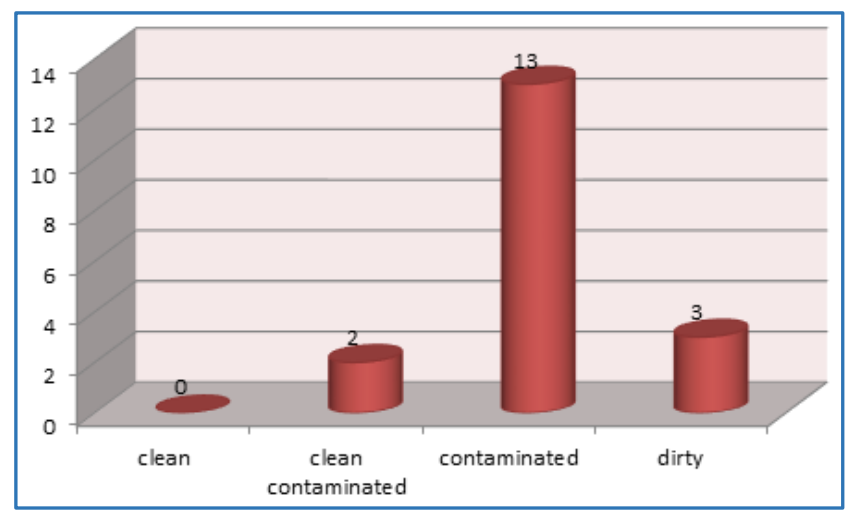

Incidence of SSI was nil [0\%] in the clean wound, 2 [11.5\%] in the clean contaminated type, 13 [72\%] in contaminated type and $3[16.5 \%]$ in dirty type.

\section{Organisms in SSI}

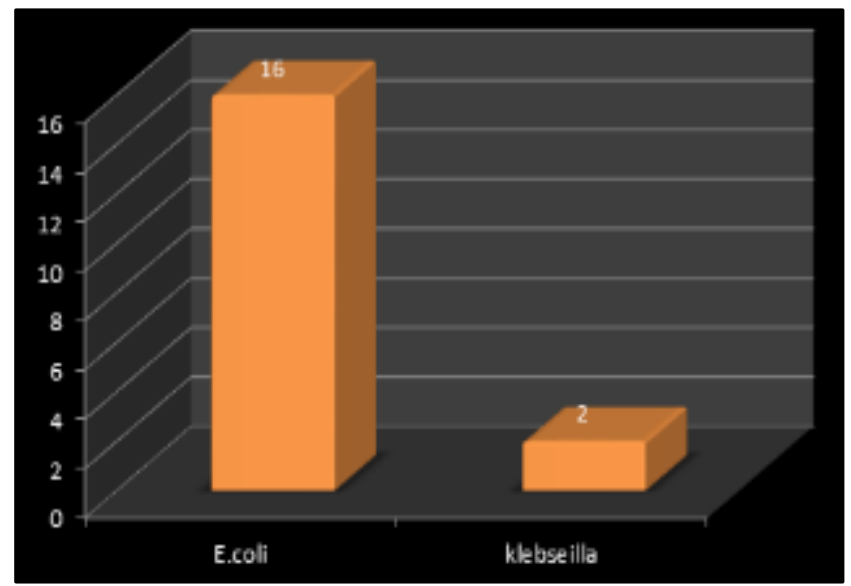

Escherichia coli was present in $89 \%$ and Klebsiella spp. in $11 \%$.

\section{Incidence of complications system wise}

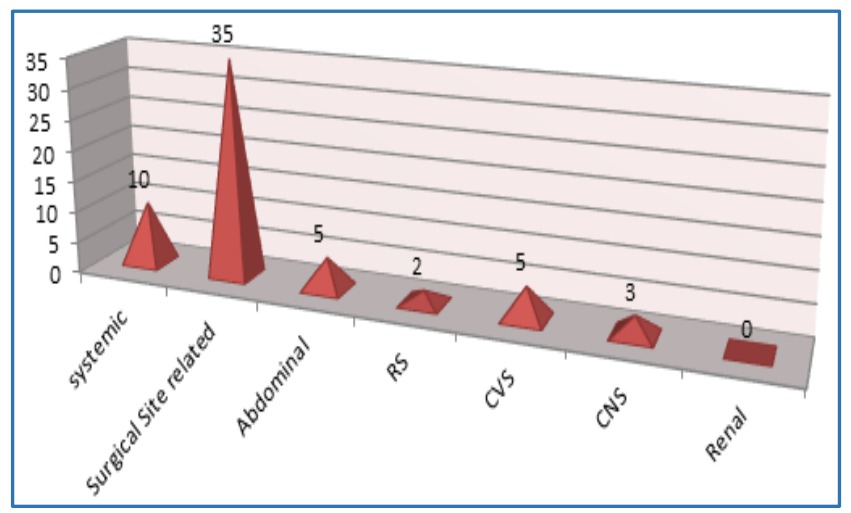

In our study surgical site related complications such as SSI, dehiscence burst abdomen, stitch abscess, fistula and hernia constituted the major postoperative complications. Haematological and systemic causes constituted the next which included surgical site bleeding, haematoma, fever and DVT. Next in frequency was cardiovascular complications and abdominal complications. Abdominal complications included subdiaphragmatic abscess and postoperative ileus.

Cardiovascular complications included hypotension, arrhythmia and myocardial ischemia. In our study, myocardial ischemia in the postoperative period was associated with $100 \%$ mortality.12,13,17 Central nervous system complication, which was present in our study was delirium. Most common association which was found in our study was between delirium tremens and postoperative delirium.

Respiratory complication atelectasis was present in 2 patients and was associated with upper abdominal surgery, mostly involving the liver or spleen. Conservative treatment with bronchodilators, physiotherapy and cough with a pillow placed over the incision site cleared it.

\section{Disease progress in patients with Haemoglobin $<9$ gram}

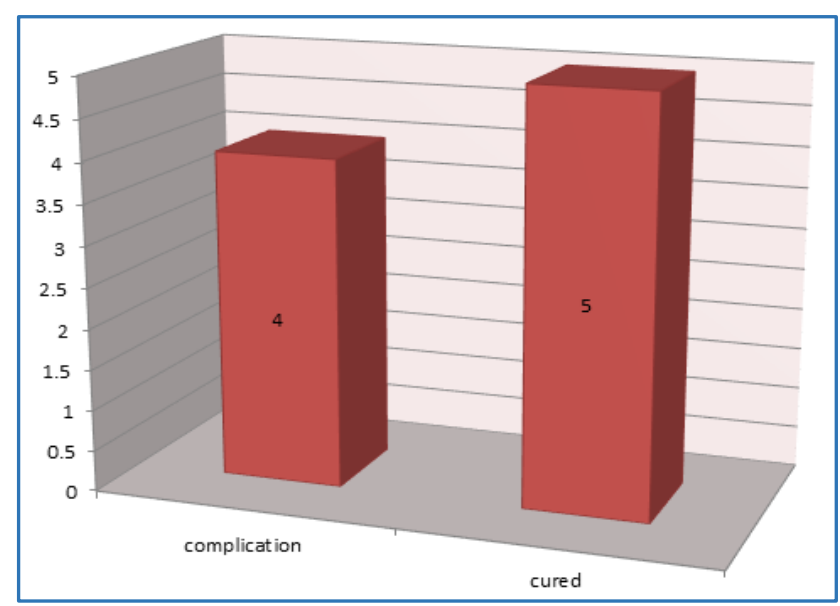

In $9 \%$ of patients, where haemoglobin level was less than $9 \mathrm{gm} \%$, 4 were cured and 5 had complications in the postoperative period. Higher percentage of cure in the anaemia group is due to the fact that in blunt injury abdomen, there is acute loss of blood, which returns to normal in the 
postoperative period after taking control of the haemorrhage and postoperative and intraoperative blood transfusion. ${ }^{18}$

\section{Relation of hypoalbuminaemia with complications}

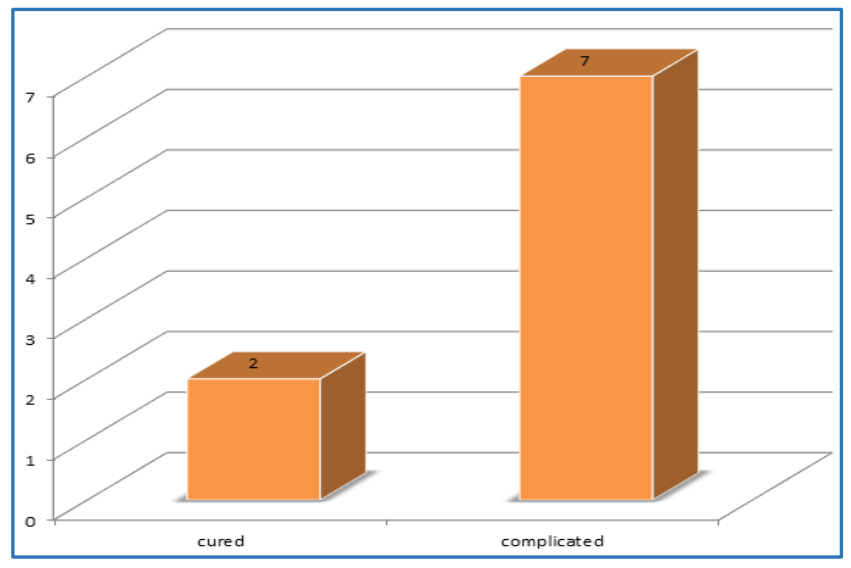

Out of 100 patients 9 patients had hypoalbuminaemia, out of which 2 got cured and remaining 7 patients had enormous complications such as SSI [ 6 out of 7 patients], fever [2 out of 7 patients], wound dehiscence [5 out of 7 patients] ileus [3 out of 7], delirium [2 out of 7 patients], subdiaphragmatic abscess [one patient], burst abdomen [one patient], stitch abscess in one patient and incisional hernia in one patient.

Surgical site related complications were more in hypoalbuminaemia than any other individual parameter. Correction of albumin with $20 \%$ albumin related to an earlier recovery and discharge.

\section{Complication Management}

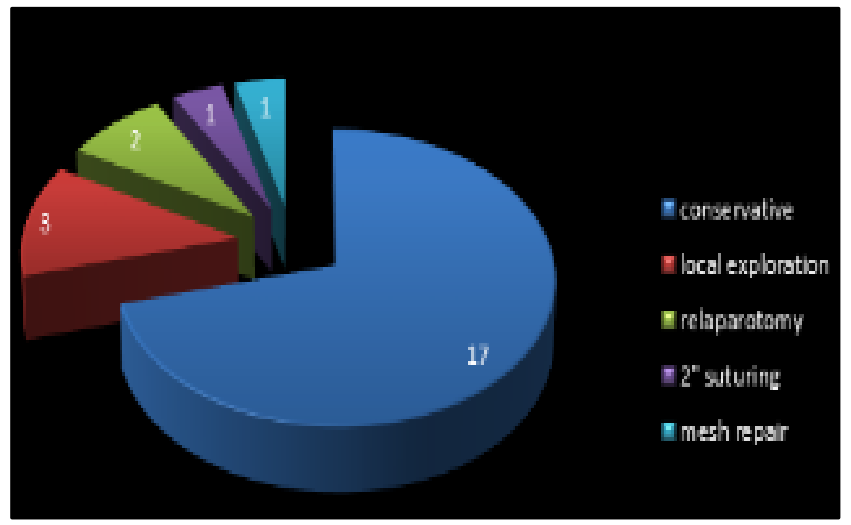

\begin{tabular}{|c|c|}
\hline Conservative & 17 \\
\hline Local exploration & 3 \\
\hline Re-laparotomy & 2 \\
\hline 2" suturing & 1 \\
\hline Mesh repair & 1 \\
\hline
\end{tabular}

In our study of 100 cases complication occurred in 27 patients, out of which 3 died in the postoperative period and the remaining patients were subjected to treatment according to their complications. Conservative management proved ideal in 17 [74\%] patients and there was no need for further surgical management. In the remaining local exploration for stitch abscess proved ideal and only in 2 cases emergency relaparotomy was indicated. There was no mortality in the relaparotomy group. ${ }^{19,20}$
Secondary suturing and incisional hernia repair was done for the remaining 2 cases. Secondary suturing was done under local anaesthesia and with 3-0 Prolene. Mesh repair of the incision hernia was done after 6 months after improving the general condition and by placing a Vicryl-Prolene mesh.

\section{DISCUSSION}

In our study of hundred laparotomies, laparotomy was done more common in the 31 to 40 years' age group to be followed by 41 to 50 years' group. Perforation of hollow viscus was the most common cause for laparotomy, whereas in above $60 \mathrm{yrs}$ intestinal obstruction secondary to malignancy was relatively more common.

The sex ratio male-female of emergency laparotomy was found to be $4: 1$. Males outnumbered the females due to increased outdoor activities, smoking and alcohol. Duodenal perforation was more common in males. Overview of the causes for emergency laparotomy revealed $64 \%$ due to hollow viscus perforation next to follow was intestinal obstruction $23 \%$, solid organ injury $11 \%$ and mesenteric ischemia with high mortality (80-90\%).

Solid organ injury was found only in males in study and the intake of alcohol by males predisposes the patient to liver abscess rupture. According to Cooper et al. $8 \%$ of cases of acute abdomen have solid organ injury and $83 \%$ were due to blunt trauma.[96] Looking on the specific aetiology of acute abdomen duodenal perforation was the single most common cause of acute abdomen in our study with $29 \%$. Intestinal obstruction constituted $23 \%$ and to be followed with perforated appendicitis in $14 \%$.

Gastric perforation constituted $10 \%$ for emergency laparotomy. Liver laceration following blunt injury abdomen with marked hypotension constituted $5 \%$. Ileal perforation, sigmoid growth and splenic laceration following blunt injury constituted $4 \%$ of cases respectively. Sigmoid growth was more common in age group greater than 60 yrs.[21]

Other rare causes such as bladder rupture, caecal growth, jejunal perforation and laceration, ruptured liver abscess, mesenteric ischemia and sigmoid volvulus constituted $2 \%$ of cases respectively. Diverticulitis was rare with one case requiring surgery emergently.

In our study, $73 \%$ of patients got discharged without complications and $3 \%$ died due to postoperative complications and the remaining $24 \%$ of cases developed postoperative complications. Among the $24 \%$ of patients who developed complications, SSI accounted for $66 \%$. SSI also predisposed the patient to other complications such as wound dehiscence, burst abdomen and incisional hernia. Most common organism causing SSI was E. coli.

Wound dehiscence was present in $33 \%$ and it was always predisposed by SSI. Fever was present in $26 \%$, stitch abscess and MI constituted $22 \%$ with high mortality in postoperative MI. Paralytic ileus was present in $15 \%$, which were treated conservatively. Atelectasis was the pulmonary complication seen in our setup undergoing upper abdominal surgery. In one case of fistula, the organism was again E. coli, which responded to conservative management.

Delirium was seen in alcoholic patients undergoing emergency laparotomy in the $2^{\text {nd }}$ or $3^{\text {rd }}$ day. Bleeding from wound, haematoma, DVT, subdiaphragmatic abscess and burst abdomen were the least causes. 
In our prospective study $76 \%$ was contaminated type, $17 \%$ clean type, $4 \%$ clean and $3 \%$ dirty. Incidence of SSI in clean wound was nil, $11.5 \%$ in clear contaminated, $16.5 \%$ in dirty, $72 \%$ in contaminated type. As a whole surgical site related complication outnumbered other complications. Patients with hypoalbuminaemia had postoperative complications, more of which interfered with wound healing resulting in gaping, burst abdomen and incisional hernia. Of the total complications excluding the death 17 patients were treated conservatively, 3 required local exploration under local anaesthesia. Two patients required re-laparotomy, secondary suturing in one patient and mesh repair were done for incisional hernia after 6 months of previous surgery.

\section{CONCLUSION}

- Acute abdomen was more common among 31 to 40 years' group.

- Male-to-female ratio was 4:1.

- Hollow viscus perforation was the most common cause for emergency laparotomy.

- Duodenal perforation was the single most common cause for emergency laparotomy.

- Diverticulitis was the least common cause for emergency laparotomy.

- $\quad$ Postoperative complication rate was $27 \%$.

- Postoperative mortality was $3 \%$.

- $\quad$ SSI is the most postoperative complication.

- E. coli was the most common cause for SSI.

- Postoperative myocardial ischemia had higher mortality.

- Contaminated wound was the most common type in emergency laparotomies.

- Hypoalbuminaemia results in postoperative wound complications.

- $20 \%$ albumin in the postoperative period resulted in early wound healing and reduced hospital stay.

- Postoperative complications can be managed conservatively in $74 \%$.

- Surgical management needed only in $26 \%$ of complications.

\section{BIBLIOGRAPHY}

1. Mudge M, Hughes LE. Incisional hernia: a 10-year prospective study of incidence and attitudes. Br J Surg 1985;72:70-71.

2. Thompson JB, MacLean KF, Coller FA. Role of the transverse abdominal incision and early ambulation in the reduction of postoperative complications. Arch Surg 1949;59:1267-1277.

3. Blomstedt B, Welin-Berger T. Incisional hernias. A comparison between midline, oblique and transrectal incisions. Acta Chir Scand 1972;138:275-278.

4. Cox PJ, Ausobsky JR, Ellis H, et al. Towards no incisional hernias: lateral paramedian versus midline incisions. J R Soc Med 1986;79:711-712.

5. Ali J, Khan TA. The comparative effects of muscle transection and median upper abdominal incisions on postoperative pulmonary function. Surg Gynecol Obstet 1979;148:863-866.
6. Douketis JD, Berger PB, Dunn AS, et al. The perioperative management of antithrombotic therapy: American College of Chest Physicians Evidence-Based Clinical Practice Guidelines (8th Edition). Chest 2008;133:299S339S.

7. Heller L, Levin SL, Butler CE. Management of abdominal wound dehiscence using vacuum-assisted closure in patients with compromised healing. Am J Surg 2006;191:165-172.

8. Mangram AJ, Horan TC, Pearson ML, et al. Guideline for prevention of surgical site infection, 1999. Hospital Infection Control Practices Advisory Committee. Infect Control Hosp Epidemiol 1999;20:250-278.

9. Awad SS, Elhabash SI, Lee L, et al. Increasing incidence of methicillin-resistant staphylococcus aureus skin and soft-tissue infections: reconsideration of empiric antimicrobial therapy. Am J Surg 2007;194:606-610.

10. National Nosocomial Infections Surveillance (NNIS) System Report, Data Summary from January 1992-June 2001, issued August 2001. Am J Infect Control 2001;29:404-421.

11. Culver DH, Horan TC, Gaynes RP, et al. Surgical wound infection rates by wound class, operative procedure and patient risk index. National Nosocomial Infections Surveillance System. Am J Med 1991;91:152S-157S.

12. Anderson DJ, Kaye KS, Classen D, et al. Strategies to prevent surgical site infections in acute care hospitals. Infect Control Hosp Epidemiol 2008;29 (Suppl 1):S51-S61.

13. Heit JA, Silverstein MD, Mohr DN, et al. Risk factors for deep vein thrombosis and pulmonary embolism: a population-based case-control study. Arch Intern Med 2000;160:809-815.

14. Goldhaber SZ. Echocardiography in the management of pulmonary embolism. Ann Intern Med 2002;136:691700 .

15. Geerts WH, Bergqvist D, Pineo GF, et al. Prevention of venous thromboembolism: American College of Chest Physicians Evidence-Based Clinical Practice Guidelines (8th Edition). Chest 2008;133:381S-453S.

16. Eagle KA, Berger PB, Calkins H, et al. ACC/AHA Guideline Update for Perioperative Cardiovascular Evaluation for Noncardiac Surgery-Executive Summary. A report of the American College of Cardiology/American Heart Association Task Force on Practice Guidelines (Committee to Update the 1996 Guidelines on Perioperative Cardiovascular Evaluation for Noncardiac Surgery). Anesth Analg 2002;94:1052-1064.

17. Almansaseer $Y$, Mukherjee D, Kline-Rogers EM, et al. Implementation of the ACC/AHA guidelines for preoperative cardiac risk assessment in a general medicine preoperative clinic: improving efficiency and preserving outcomes. Cardiology 2005;103:24-29.

18. Polanczyk CA, Goldman L, Marcantonio ER, et al. Supraventricular arrhythmia in patients having noncardiac surgery: clinical correlates and effect on length of stay. Ann Intern Med 1998;129:279-285.

19. Kirkpatrick AW, Balogh Z, Ball CG, et al. The secondary abdominal compartment syndrome: iatrogenic or unavoidable? J Am Coll Surg 2006;202:668-679. 
20. Simon TL, Alverson DC, AuBuchon J, et al. Practice parameter for the use of red blood cell transfusions: developed by the Red Blood Cell Administration Practice Guideline Development Task Force of the College of American Pathologists. Arch Pathol Lab Med 1998;122:130-138.
21. Uzunkoy A, Akinci OF, Coskun A, et al. Effects of antiadhesive agents on the healing of intestinal anastomosis. Dis Colon Rectum 2000;43:370-375. 